\title{
Influence of an Inclined Magnetic Field and Rotation on the Peristaltic Flow of a Micropolar Fluid in an Inclined Channel
}

\author{
Ajaz Ahmad Dar' ${ }^{1}$ and K. Elangovan ${ }^{2}$ \\ ${ }^{1}$ Department of Mathematics, Annamalai University, Annamalai Nagar 608 002, India \\ ${ }^{2}$ Mathematics Section, FEAT, Annamalai University, Annamalai Nagar 608 002, India \\ Correspondence should be addressed to Ajaz Ahmad Dar; aijaz855141@gmail.com
}

Received 22 April 2016; Accepted 18 August 2016

Academic Editor: Salvatore Magazu

Copyright (c) 2016 A. Ahmad Dar and K. Elangovan. This is an open access article distributed under the Creative Commons Attribution License, which permits unrestricted use, distribution, and reproduction in any medium, provided the original work is properly cited.

\begin{abstract}
This present article deals with the interaction of both rotation and inclined magnetic field on peristaltic flow of a micropolar fluid in an inclined symmetric channel with sinusoidal waves roving down its walls. The highly nonlinear equations are simplified by adopting low Reynolds number and long wavelength approach. The analytical and numerical solutions for axial velocity, spin velocity, volume flow rate, pressure gradient, pressure rise per wavelength, and stream function have been computed and analyzed. The quantitative effects of various embedded physical parameters are inspected and displayed graphically with fussy prominence. Pressure rise, frictional forces, and pumping phenomenon are portrayed and characterized graphically.
\end{abstract}

\section{Introduction}

Since the pioneer work done by Latham [1], several investigators have discussed the peristaltic flow because of its enormous applications in physiological fluids, biological systems, and engineering [2-6]. In particular, it incorporates the transport of urine from kidney to bladder, development of chyme in the gastrointestinal tract, transport of spermatozoa in the ducts efferent of the male reproductive tract, development of ovum in the female fallopian tube, vaso-movement of little veins, bile from gallbladder into the duodenum, and the fetus transport in nonpregnant uterus. Improper peristalsis is the wellspring of neurotic transport of microscopic organisms and thrombus improvement of blood and sterility in human uterus [7]. Peristaltic component likewise finds numerous applications in biorestorative frameworks including roller and finger pumps, heart-lung machine, blood pump machine, and dialysis machine.

The micropolar fluids are generally characterized as isotropic, polar liquids in which contortion of the particles is disregarded. Because of its unfussiness, the model of micropolar liquid is by and large utilized for elucidation of genuine liquids with inward structure. Micropolar liquid is a unique sort of non-Newtonian liquid with miniaturized scale structure which fits in with a class of liquids with nonsymmetrical stress tensor alluded to as polar liquids. Physically, it suggests liquids including unevenly arranged particles suspended in a gooey medium. Such a liquid can support couple stresses and body couples and also uncovers microrotational and microinertial properties. It is all around acknowledged that various organic liquids carry on like suspensions of deformable or unbending particles in a Newtonian liquid. Blood, for instance, is a suspension of red blood cells, white blood cells, and platelets in plasma and cervical natural fluid is a suspension of vast scale particles in water like fluid. The hypothesis of micropolar liquids was figured first by Eringen [8]. Mekheimer [9] studied the effect of induced magnetic field on the peristaltic flow of a magnetomicropolar fluid. Moreover, Pandey and Chaube [10] investigated the effect of external magnetic field on the peristaltic flow of a micropolar fluid through a porous medium. 
Magnetic fields influence many natural and man-made flows. Magnetic fields are used to heat, pump, stir, and levitate liquid metals in industries. Magnetohydrodynamics (MHD) deals with the study of electrically conducting fluid under the influence of electromagnetic field. MHD is the science which deals with the motion of conducting fluids in the presence of a magnetic field. The motion of the conducting fluid across the magnetic field generates electric currents which change the magnetic field, and the action of the magnetic field on these currents gives rise to mechanical forces which modify the flow of the fluid [11]. The effect of moving magnetic field on blood flow was studied by Sud et al. [12], and they observed that the effect of suitable moving magnetic field accelerates the speed of blood. Linked with cancer, multiple sclerosis, miscarriage, and a host of other devastating diseases, electromagnetic fields are a serious modern concern. In order to avoid these serious health problems, electromagnetic shielding must be practised. Wlodarczyk et al. [13] investigated the dielectric properties of glassy disaccharides for electromagnetic interference shielding application. The effect of electromagnetic fields on human neuronallike cells vibration bands in mid-infrared region has been examined by Calabrò et al. [14]. Calabrò [15] investigated the effects of electromagnetic fields on cells and protein structure.

Different examiners have concentrated on peristaltic stream issues in diverse geometries amid the most recent five years. Hayat et al. [16] have concentrated on the peristaltic flow of Maxwell fluid in an asymmetric channel by using perturbation method. Impact of attractive field on catching through peristaltic movement to sum up Newtonian liquid in a channel has been inspected by Abd El Naby et al. [17]. Mishra and Rao [18] have examined the stream in hilter kilter channel created by peristaltic waves engendering on the dividers with diverse hauls and stages. Srinivas and Kothandapani [19] investigated the effects of heat and mass transfer on peristaltic transport in a permeable space with grumble walls. Nadeem and Akbar [20] studied the peristaltic flow of an incompressible MHD Newtonian fluid in a vertical annulus. Abd-Alla et al. [21] investigated the effects of the rotation, magnetic field, and initial stress on peristaltic motion of a micropolar fluid. Mahmoud et al. [22] examined the impacts of revolution on wave movement through tube shaped borne in a micropolar permeable medium. Peristaltic pumping of a micropolar liquid in a tube has been explored by Srinivasacharya et al. [23]. Recently, Mohanty et al. [24] investigated heat and mass transfer effect of micropolar fluid over a stretching sheet through porous media. Abd-Alla et al. [25-28] researched the impacts of the rotation on a nonhomogeneous boundless barrel of orthotropic material. They also studied the influences of rotation, magnetic field, initial stress, and gravity on Rayleigh waves in a homogeneous orthotropic elastic half-space. As of late, numerous specialists have examined the impact of slanted channel and slanted magnetic field on peristaltic stream of a micropolar liquid [29-33].

The aim of the present study is to investigate inclination effects of magnetic field and inclined channel on the peristaltic flow of a micropolar fluid. Assumptions of low

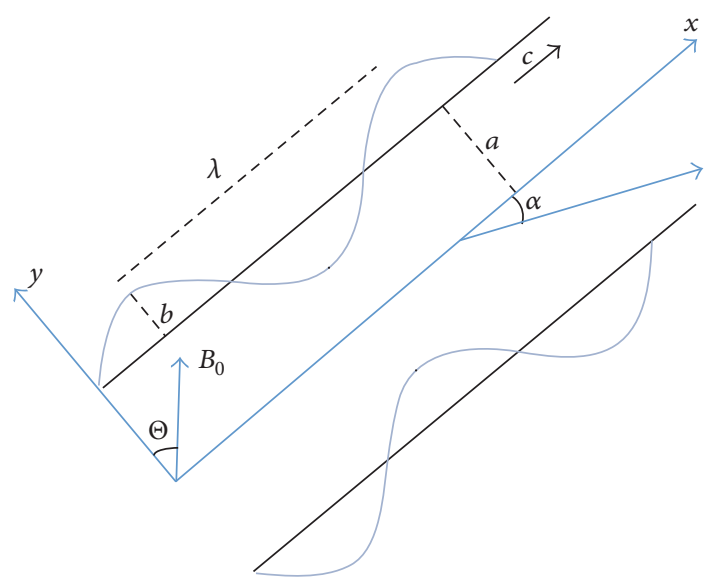

Figure 1: Geometry of the problem.

Reynolds number and long wavelength are employed. The exact expressions for axial velocity, spin velocity, stream function, and pressure rise per wavelength are obtained. The pertinent constraints have been noticed pictorially.

\section{Mathematical Formulation}

Consider the flow of an incompressible, electrically conducting micropolar fluid in an inclined channel in presence of an inclined magnetic field and rotation. The fluid fills a twodimensional channel of nonuniform thickness. Sinusoidal waves of constant speed $(c)$ propagate along the channel boundaries. The plates of the channel are assumed to be electrically insulated. We choose a rectangular coordinate system for the channel with $x$ along center-line in the direction of wave propagation and $y$ transverse to it.

The geometry of the channel walls (as shown in Figure 1) is given by

$$
h^{\prime}(x, t)=a+b \sin \frac{2 \pi}{\lambda}\left(X^{\prime}-c t^{\prime}\right)
$$

where $a$ is the half width of the channel, $b$ is the amplitude, $\lambda$ is the wavelength, $c$ is the wave speed, and $t^{\prime}$ is the time.

The flow is unsteady in the laboratory frame $\left(X^{\prime}, Y^{\prime}\right)$ whereas it is steady if observed in the coordinate system $\left(x^{\prime}, y^{\prime}\right)$, termed as a wave frame, moving with velocity $c$. The transformation from the fixed frame of reference $\left(X^{\prime}, Y^{\prime}\right)$ to the wave frame of reference $\left(x^{\prime}, y^{\prime}\right)$ is given by

$$
\begin{aligned}
& x^{\prime}=X^{\prime}-c t^{\prime}, \\
& y^{\prime}=Y^{\prime}, \\
& u^{\prime}=U^{\prime}-c, \\
& v^{\prime}=V^{\prime} .
\end{aligned}
$$


In absence of body forces and body couple, the governing equations for the flow in wave frame of reference are given by

$$
\begin{aligned}
& \frac{\partial u^{\prime}}{\partial x^{\prime}}+\frac{\partial v^{\prime}}{\partial y^{\prime}}=0 \\
& \rho\left(u^{\prime} \frac{\partial u^{\prime}}{\partial x^{\prime}}+v^{\prime} \frac{\partial u^{\prime}}{\partial y^{\prime}}\right)-\rho\left(\Omega^{2} u^{\prime}+2 \Omega \frac{\partial v^{\prime}}{\partial t^{\prime}}\right) \\
& =-\frac{\partial p^{\prime}}{\partial x^{\prime}}+(\mu+k)\left(\frac{\partial^{2} u^{\prime}}{\partial x^{\prime 2}}+\frac{\partial^{2} u^{\prime}}{\partial y^{\prime 2}}\right)+k \frac{\partial w^{\prime}}{\partial y^{\prime}} \\
& -\sigma B_{0}^{2} \cos \Theta\left(\cos \Theta\left(u^{\prime}+c\right)-v^{\prime} \sin \Theta\right) \\
& +\rho g \sin \alpha, \\
& \rho\left(u^{\prime} \frac{\partial v^{\prime}}{\partial x^{\prime}}+v^{\prime} \frac{\partial v^{\prime}}{\partial y^{\prime}}\right)+\rho\left(-\Omega^{2} v^{\prime}+2 \Omega \frac{\partial u^{\prime}}{\partial t^{\prime}}\right) \\
& =-\frac{\partial p^{\prime}}{\partial y^{\prime}}+(\mu+k)\left(\frac{\partial^{2} v^{\prime}}{\partial x^{\prime 2}}+\frac{\partial^{2} v^{\prime}}{\partial y^{\prime 2}}\right)-k \frac{\partial w^{\prime}}{\partial x^{\prime}} \\
& +\sigma B_{0}^{2} \sin \Theta\left(\cos \Theta\left(u^{\prime}+c\right)-v^{\prime} \sin \Theta\right) \\
& -\rho g \cos \alpha, \\
& \rho j^{\prime}\left(u^{\prime} \frac{\partial w^{\prime}}{\partial x^{\prime}}+v^{\prime} \frac{\partial w^{\prime}}{\partial y^{\prime}}\right) \\
& =-2 k w^{\prime}+\gamma\left(\frac{\partial^{2} w^{\prime}}{\partial x^{\prime 2}}+\frac{\partial^{2} w^{\prime}}{\partial y^{\prime 2}}\right)+k\left(\frac{\partial v^{\prime}}{\partial x^{\prime}}-\frac{\partial u^{\prime}}{\partial y^{\prime}}\right),
\end{aligned}
$$

where $u^{\prime}$ and $v^{\prime}$ are the velocity components in the $x^{\prime}$ and $y^{\prime}$ directions, respectively, $\rho$ is the density of the fluid, $p^{\prime}$ is the pressure, $\mu$ is the viscosity constant of the classical fluid dynamics, $\sigma$ is the electrical conductivity, $B_{0}$ is the strength of the magnetic field, $g$ is acceleration due to gravity, $\Theta$ is inclination angle of the magnetic field parameter, $\alpha$ is inclination angle of the channel, $w$ is the microrotation velocity component in the direction normal to both the $x^{\prime}$ and $y^{\prime}$ axes, $j^{\prime}$ is the microinertia density, $k$ is the microrotation parameter, and $\gamma$ is the spin-gradient viscosity coefficient $[34,35]$.

Now we introduce the nondimensional variables and parameters as follows:

$$
\begin{aligned}
& x=\frac{x^{\prime}}{\lambda}, \\
& y=\frac{y^{\prime}}{a}, \\
& u=\frac{u^{\prime}}{c}, \\
& v=\frac{v^{\prime}}{\delta c},
\end{aligned}
$$

$$
\begin{aligned}
\delta & =\frac{a}{\lambda}, \\
t & =\frac{c t^{\prime}}{\lambda}, \\
p & =\frac{p^{\prime} a^{2}}{c \lambda \mu}, \\
j & =\frac{j^{\prime}}{a^{2}}, \\
w & =\frac{a w^{\prime}}{c}, \\
h & =\frac{h^{\prime}}{a}, \\
M & =\frac{\sqrt{\frac{\sigma}{\mu}}}{\sqrt{a}}, \\
R_{e} & =\frac{\rho c a}{\mu}, \\
F_{r} & =\frac{c^{2}}{g a}, \\
h & =1+\phi \sin 2 \pi(x-t), \\
\phi &
\end{aligned}
$$

where $M$ is the Hartman number, $R_{e}$ is the Reynolds number, $F_{r}$ is the Froude number, and $\delta$ is the wave number.

Using the nondimensional variables and parameters given above in (4), (5), and (6), we get the modified equations as

$$
\begin{aligned}
R_{e} \delta( & \left.u \frac{\partial u}{\partial x}+v \frac{\partial u}{\partial y}\right)-\left(\frac{\rho \Omega^{2} a^{2}}{\mu} u+\frac{2 \Omega \delta R_{e}}{\lambda} \frac{\partial v}{\partial t}\right) \\
= & -\frac{\partial p}{\partial x}+\frac{(\mu+k)}{\mu}\left(\delta^{2} \frac{\partial^{2} u}{\partial x^{2}}+\frac{\partial^{2} u}{\partial y^{2}}\right)+\frac{k}{\mu} \frac{\partial w}{\partial y} \\
& -M^{2} \cos \Theta((u+1) \cos \Theta-v \delta \sin \Theta) \\
& +\frac{R_{e}}{F_{r}} \sin \alpha, \\
R_{e} \delta^{3} & \left(u \frac{\partial v}{\partial x}+v \frac{\partial v}{\partial y}\right)+\left(-\frac{\rho \delta^{2} \Omega^{2} a^{2}}{\mu} v+2 \Omega \delta^{2} R_{e} \frac{\partial u}{\partial t}\right) \\
= & -\frac{\partial p}{\partial y}+\frac{(\mu+k)}{\mu} \delta^{2}\left(\delta^{2} \frac{\partial^{2} v}{\partial x^{2}}+\frac{\partial^{2} v}{\partial y^{2}}\right)-\delta^{2} \frac{k}{\mu} \frac{\partial w}{\partial x} \\
& +M^{2} \delta \sin \Theta((u+1) \cos \Theta-v \delta \sin \Theta) \\
& -\delta \frac{R_{e}}{F_{r}} \cos \alpha,
\end{aligned}
$$




$$
\begin{aligned}
\operatorname{\rho acj} \delta & \left(u \frac{\partial w}{\partial x}+v \frac{\partial w}{\partial y}\right) \\
= & -2 k w+\frac{\gamma}{a^{2}}\left(\delta^{2} \frac{\partial^{2} w}{\partial x^{2}}+\frac{\partial^{2} w}{\partial y^{2}}\right) \\
& +k\left(\delta^{2} \frac{\partial v}{\partial x}-\frac{\partial u}{\partial y}\right) .
\end{aligned}
$$

Using the long wavelength approximation and neglecting the wave number along with low Reynolds number, one can find from (8) that

$$
\begin{aligned}
& -(1-N) \frac{\partial p}{\partial x}+\frac{\partial^{2} u}{\partial y^{2}}+N \frac{\partial w}{\partial y} \\
& -(1-N) M^{2} \cos ^{2} \Theta(u+1)+(1-N) \frac{R_{e}}{F_{r}} \sin \alpha \\
& \quad+D u=0, \\
& \frac{\partial p}{\partial y}=0, \\
& -2 w-\frac{\partial u}{\partial y}+\left(\frac{2-N}{m^{2}}\right) \frac{\partial^{2} w}{\partial y^{2}}=0,
\end{aligned}
$$

where $D=\rho \Omega^{2} a^{2} /(\mu+k), N=k /(\mu+k)$ is the coupling number $(0 \leq N \leq 1)$, and $m^{2}=a^{2} k(2 \mu+k) / \gamma(\mu+k)$ is the micropolar parameter.

\section{Rate of Volume Flow and Boundary Conditions}

The instantaneous volume flow rate in the fixed frame is given as

$$
Q\left(X^{\prime}, t^{\prime}\right)=\int_{0}^{H} U^{\prime}\left(X^{\prime}, Y^{\prime}, t^{\prime}\right) d Y^{\prime}
$$

where $H$ is a function of $X^{\prime}$ and $t^{\prime}$.

The rate of volume flow in the wave frame can be expressed as

$$
q\left(x^{\prime}\right)=\int_{0}^{H} u^{\prime}\left(x^{\prime}, y^{\prime}\right) d y^{\prime}
$$

where $H$ is a function of $x^{\prime}$ alone. Equations (2), (12), and (13) yield

$$
Q\left(X^{\prime}, t^{\prime}\right)=q\left(x^{\prime}\right)+c H\left(X^{\prime}, t^{\prime}\right)
$$

The time averaged mean flow rate over a period $T$ at a fixed position $X^{\prime}$ is expressed as

$$
\bar{Q}\left(X^{\prime}\right)=\frac{1}{T} \int_{0}^{T} Q\left(X^{\prime}, t^{\prime}\right) d t^{\prime} .
$$

Using (14) into (15), we get

$$
\bar{Q}\left(X^{\prime}\right)=q\left(x^{\prime}\right)+a c .
$$

The dimensionless mean flow rates $\widetilde{Q}$ (in the laboratory frame) and $\theta$ (in the wave frame) are defined as

$$
\begin{aligned}
& \widetilde{Q}=\frac{\bar{Q}\left(X^{\prime}\right)}{a c}, \\
& \theta=\frac{q\left(x^{\prime}\right)}{a c} .
\end{aligned}
$$

By using (17) in (16), we get

$$
\widetilde{Q}=\theta(x)+1,
$$

where

$$
\theta(x)=\int_{0}^{h} u(x, y) d y .
$$

The dimensionless boundary conditions in the wave frame of reference are given as

$$
\begin{aligned}
& u(x, y)=-1 \quad \text { for } y= \pm h(x), \\
& w(x, y)=0 \quad \text { for } y= \pm h(x) .
\end{aligned}
$$

\section{Solution of the Problem}

From (10) it is clear that $p$ is independent of $y$. Therefore $\partial p / \partial x=d p / d x$. Differentiating (11) with respect to $y$, we get

$$
\frac{\partial^{2} u}{\partial y^{2}}=-2 \frac{\partial w}{\partial y}+\left(\frac{2-N}{m^{2}}\right) \frac{\partial^{3} w}{\partial y^{3}} .
$$

Upon making use of (21) in (9) we get

$$
\begin{aligned}
u & =\frac{1}{(1-N) M^{2} \cos ^{2} \Theta-D}\left\{\left(\frac{2-N}{m^{2}}\right) \frac{\partial^{3} w}{\partial y^{3}}\right. \\
& -(2-N) \frac{\partial w}{\partial y} \\
& \left.-(1-N)\left(\frac{d p}{d x}+M^{2} \cos ^{2} \Theta-\frac{R_{e}}{F_{r}} \sin \alpha\right)\right\} .
\end{aligned}
$$

Using value of $u$ from (22) in (11), we get

$$
\begin{array}{r}
\frac{\partial^{4} w}{\partial y^{4}}-\left((1-N) M^{2} \cos ^{2} \Theta-D+m^{2}\right) \frac{\partial^{2} w}{\partial y^{2}} \\
+\frac{2 m^{2}\left((1-N) M^{2} \cos ^{2} \Theta-D\right)}{2-N} w=0
\end{array}
$$


whose general solution is

$$
\begin{aligned}
w= & A(x) \cosh \left(\theta_{1} y\right)+B(x) \sinh \left(\theta_{1} y\right) \\
& +C(x) \cosh \left(\theta_{2} y\right)+D(x) \sinh \left(\theta_{2} y\right),
\end{aligned}
$$

where

$$
\begin{aligned}
& \theta_{1}=\frac{1}{\sqrt{2}} \sqrt{A_{1}-\sqrt{\left(A_{1}\right)^{2}-4 A_{2}}}, \\
& \theta_{2}=\frac{1}{\sqrt{2}} \sqrt{A_{1}+\sqrt{\left(A_{1}\right)^{2}-4 A_{2}}},
\end{aligned}
$$

where

$$
\begin{aligned}
& A_{1}=(1-N) M^{2} \cos ^{2} \Theta-D+m^{2}, \\
& A_{2}=\frac{2 m^{2}\left((1-N) M^{2} \cos ^{2} \Theta-D\right)}{2-N} .
\end{aligned}
$$

Substituting (24) into (22), we get

$$
\begin{aligned}
u= & \frac{1}{(1-N) M^{2} \cos ^{2} \Theta-D}\left\{\bigwedge _ { 1 } \left[A(x) \sinh \left(\theta_{1} y\right)\right.\right. \\
& \left.+B(x) \cosh \left(\theta_{1} y\right)\right]+\bigwedge_{2}\left[C(x) \sinh \left(\theta_{2} y\right)\right. \\
& \left.+D(x) \cosh \left(\theta_{2} y\right)\right]-(1-N)\left(\frac{d p}{d x}+M^{2} \cos ^{2} \Theta\right. \\
& \left.\left.-\frac{R_{e}}{F_{r}} \sin \alpha\right)\right\},
\end{aligned}
$$

where

$$
\begin{aligned}
& \bigwedge_{1}=(2-N) \theta_{1}\left(\frac{\theta_{1}^{2}}{m^{2}}-1\right), \\
& \bigwedge_{2}=(2-N) \theta_{2}\left(\frac{\theta_{2}^{2}}{m^{2}}-1\right) .
\end{aligned}
$$

The expressions for axial and spin velocities can be found by applying the boundary conditions from (20) into (24) and (27). The expression for axial velocity is given as

$$
\begin{aligned}
= & \frac{\left((1-N)\left(d p / d x-\left(R_{e} / F_{r}\right) \sin \alpha\right)+D\right)}{(1-N) M^{2} \cos ^{2} \Theta-D}\left(\frac{\left(m^{2}-\theta_{2}^{2}\right) \theta_{2} \tanh \left(\theta_{1} h\right)\left(\cosh \left(\theta_{2} y\right) / \cosh \left(\theta_{2} h\right)\right)+\left(\theta_{1}^{2}-m^{2}\right) \theta_{1} \tanh \left(\theta_{2} h\right)\left(\cosh \left(\theta_{1} y\right) / \cosh \left(\theta_{1} h\right)\right)}{\left(m^{2}-\theta_{2}^{2}\right) \theta_{2} \tanh \left(\theta_{1} h\right)+\left(\theta_{2}^{2}-m^{2}\right) \theta_{1} \tanh \left(\theta_{2} h\right)}\right) \\
& -\frac{\left((1-N)\left(d p / d x+M^{2} \cos ^{2} \Theta-\left(R_{e} / F_{r}\right) \sin \alpha\right)+D\right)}{(1-N) M^{2} \cos ^{2} \Theta-D}
\end{aligned}
$$

and the expression for spin velocity is given as

$w$

$$
=\frac{D m^{2}+(1-N) m^{2}\left(d p / d x-\left(R_{e} / F_{r}\right) \sin \alpha\right)}{(2-N)}\left\{\frac{\tanh \left(\theta_{2} h\right)\left(\sinh \left(\theta_{1} y\right) / \cosh \left(\theta_{1} h\right)\right)-\tanh \left(\theta_{1} h\right)\left(\sinh \left(\theta_{2} y\right) / \cosh \left(\theta_{2} h\right)\right)}{\left(m^{2}-\theta_{2}^{2}\right) \theta_{2} \tanh \left(\theta_{1} h\right)+\left(\theta_{1}^{2}-m^{2}\right) \theta_{1} \tanh \left(\theta_{2} h\right)}\right\}
$$

The stream function $(\psi)$ is given by

$$
v=-\frac{\partial \psi}{\partial x}
$$

$$
u=\frac{\partial \psi}{\partial y}
$$

Using (31) in (29), we get

$\psi$

$$
\begin{aligned}
= & \frac{\left((1-N)\left(d p / d x-\left(R_{e} / F_{r}\right) \sin \alpha\right)+D\right)}{(1-N) M^{2} \cos ^{2} \Theta-D}\left(\frac{\left(m^{2}-\theta_{2}^{2}\right) \tanh \left(\theta_{1} h\right)\left(\sinh \left(\theta_{2} y\right) / \cosh \left(\theta_{2} h\right)\right)+\left(\theta_{1}^{2}-m^{2}\right) \tanh \left(\theta_{2} h\right)\left(\sinh \left(\theta_{1} y\right) / \cosh \left(\theta_{1} h\right)\right)}{\left(m^{2}-\theta_{2}^{2}\right) \theta_{2} \tanh \left(\theta_{1} h\right)+\left(\theta_{1}^{2}-m^{2}\right) \theta_{1} \tanh \left(\theta_{2} h\right)}\right) \\
& -\frac{\left((1-N)\left(d p / d x+M^{2} \cos ^{2} \Theta-\left(R_{e} / F_{r}\right) \sin \alpha\right)+D\right)}{(1-N) M^{2} \cos ^{2} \Theta-D} y .
\end{aligned}
$$


The expression for dimensionless flow rate in the wave frame of reference is obtained by substituting (29) into (19) and is given as

$$
\theta=\frac{\left((1-N)\left(d p / d x-\left(R_{e} / F_{r}\right) \sin \alpha\right)+D\right)(F(h)-h)-(1-N) M^{2} \cos ^{2} \Theta h}{(1-N) M^{2} \cos ^{2} \Theta-D}
$$

where

$F(h)$

$$
=\frac{\left(\theta_{1}^{2}-\theta_{2}^{2}\right) \tanh \left(\theta_{1} h\right) \tanh \left(\theta_{2} h\right)}{\left(m^{2}-\theta_{2}^{2}\right) \theta_{2} \tanh \left(\theta_{1} h\right)+\left(\theta_{2}^{2}-m^{2}\right) \theta_{1} \tanh \left(\theta_{2} h\right)} .
$$

From (33) and (18), we obtain

$$
\frac{d p}{d x}=\frac{\left(M^{2} \cos ^{2} \Theta(1-N)-D\right)(\widetilde{Q}-1)+\left(M^{2} \cos ^{2} \Theta(1-N)+D\right) h-D F(h)}{(F(h)-h)(1-N)}+\frac{R_{e}}{F_{r}} \sin \alpha .
$$

The expressions for pressure rise $\Delta p$ and the frictional force $F$ over one wavelength are given by

$$
\begin{aligned}
\Delta p & =\int_{0}^{1}\left(\frac{d p}{d x}\right) d x \\
F & =\int_{0}^{1} h\left(-\frac{d p}{d x}\right) d x .
\end{aligned}
$$

\section{Numerical Results and Discussion}

In the proceeding section the quantitative effects of the various parameters involved in the results of the present flow problem have been estimated and displayed with the graphical illustration. Mathematics software Mathematica is used to evaluate the numerical solution of pressure rise and frictional forces. The variations for axial velocity $(u)$, pressure gradient $(d p / d x)$, pressure rise $(\Delta p)$, frictional forces $(F)$, spin velocity $(w)$, and stream function $(\psi)$ are outlined in Figures 2-7 individually, with the steady values $N=0.2$, $m=2, M=1, \phi=0.5, \Theta=\pi / 3$, and $\alpha=\pi / 3$.

Figures 2(a)-2(h) illustrate the variation of the value of axial velocity $(u)$ with respect to $y$ for different values of the rotation $(\Omega)$, magnetic field parameter $(M)$, coupling number $(N)$, micropolar parameter $(m)$, inclination angle of the channel $(\alpha)$, Froude number $\left(F_{r}\right)$, Reynold number $\left(R_{e}\right)$, and the inclination angle of the magnetic field parameter $(\Theta)$. From these figures it is observed that the maximum velocity is always located near the center of the channel and the velocity profiles are parabolic in all cases. It is revealed from Figures 2(a)-2(c) and 2(f) that the axial velocity decreases with increasing of rotation, magnetic field parameter, coupling number, and Froude number. It is depicted from Figure 2(d) that the axial velocity increases by increasing the micropolar parameter $(m)$. The impact of Reynold number, inclination angle of the channel, and the magnetic field parameter on the axial velocity speed is like the micropolar parameter.

Figures 3-5 describe the influence of different embedding parameters on the pressure gradient, pressure rise per wavelength, and the frictional forces. In Figures 3(a)-3(g), we have plotted pressure gradient against $x$ for different physical parameters of interest. It is observed from these figures that the pressure gradient is comparatively small in the wider part of the channel, $x \in[0,0.6]$ and $x \in$ $[0.9,1]$; that is, the flow can easily pass without applying large pressure gradient. However, in the narrow part of the channel, $x \in[0.6,0.8]$, particularly near $x=0.75$, the pressure gradient is comparatively larger; that is, larger pressure gradient is required to maintain the same flux to pass through it. From Figures 3(a)-3(c) it is depicted that the pressure gradient decreases with increasing the rotation $(\Omega)$, Froude number $\left(F_{r}\right)$, and the coupling number $(N)$. Figures 3(d)-3(e) illustrate that increasing the micropolar parameter $(m)$ and the inclination angle of the magnetic field parameter $(\Theta)$ increases the pressure gradient in the wider part of the channel, $x \in[0,0.6]$ and $x \in[0.9,1]$, while in the narrow part of the channel, $x \in[0.6,0.8]$, there is no noticeable difference. Figure 3(f) shows that the pressure gradient increases by increasing the inclination angle of the channel $(\alpha)$. From Figure 3(g) it is illustrated that the behavior of magnetic field parameter $(M)$ on the pressure gradient is quite opposite when compared with micropolar parameter $(m)$.

The variation of pressure rise $(\Delta p)$ over one wavelength, friction forces $(F)$ across one wavelength against the average volume flux $(\widetilde{Q})$ has been illustrated in Figures 4 and 5 for different values of $\Omega, M, N, m, \alpha, \Theta, F_{r}$, and $R_{e}$. It is determined that the behavior of pressure rise and volume flow rate is quite opposite. In these figures the region is divided into four parts: peristaltic pumping region $(\Delta p>0, \widetilde{Q}>0)$, retrograde pumping region $(\Delta p>0, \widetilde{Q}<0)$, augmented 


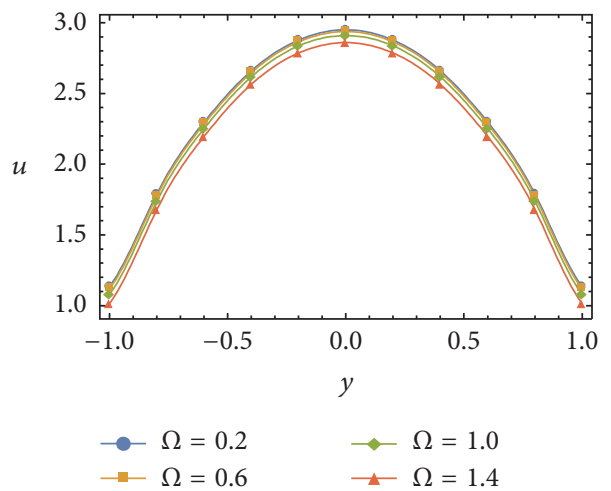

(a)

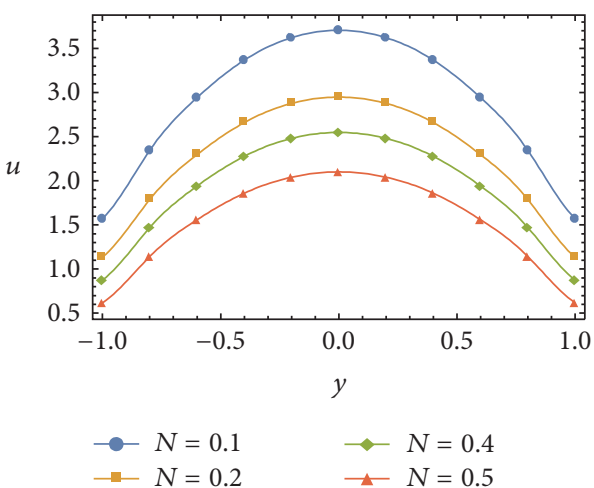

(c)

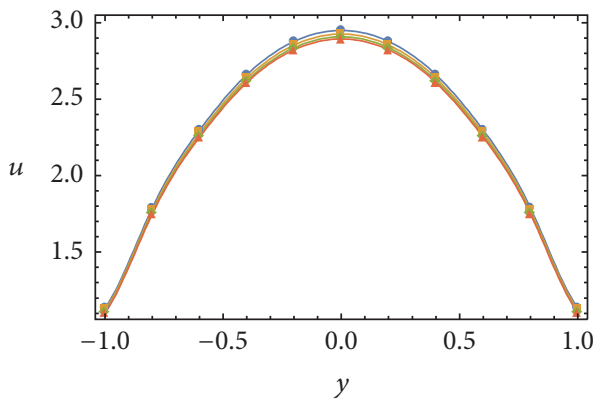

$$
\longrightarrow \alpha=\pi / 3 \quad \longrightarrow \alpha=\pi / 5
$$

(e)

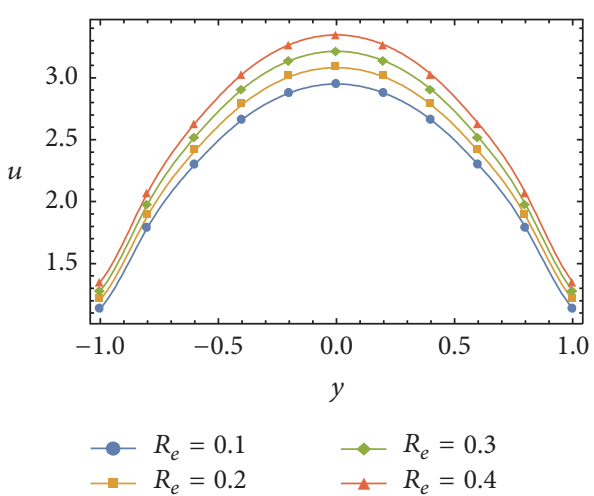

(g)

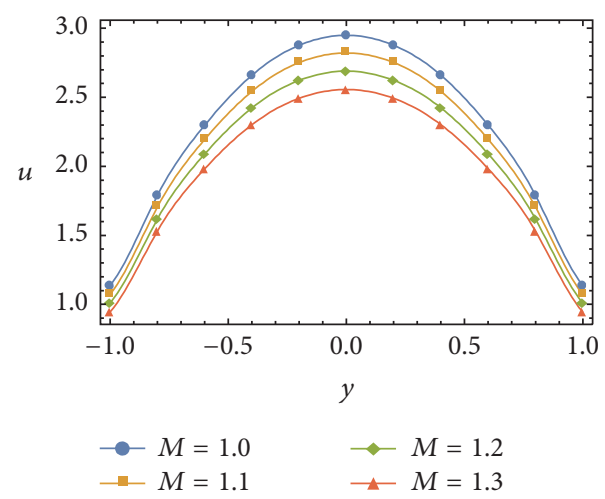

(b)

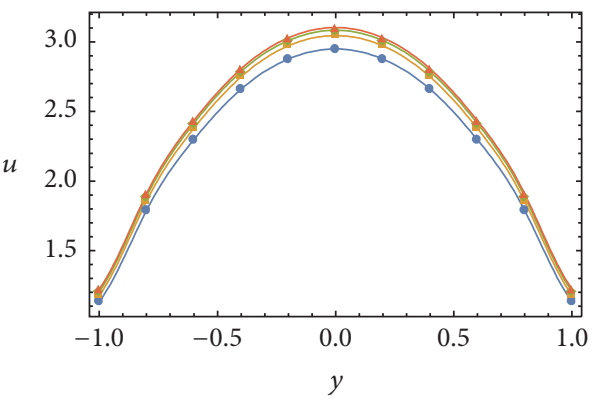

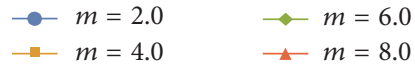

(d)

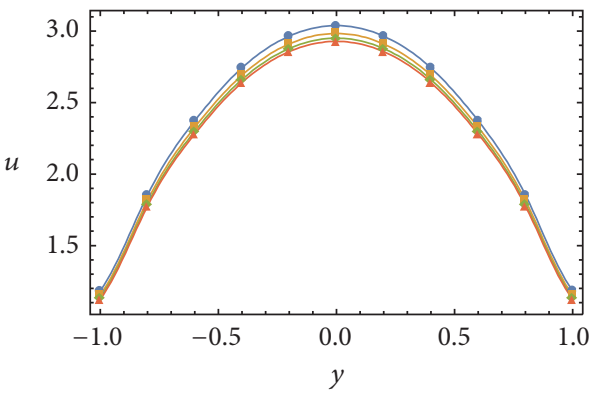

$$
\begin{aligned}
& \longrightarrow F_{r}=0.3 \quad \rightarrow \quad F_{r}=0.5 \\
& \because F_{r}=0.4 \quad \longrightarrow F_{r}=0.6
\end{aligned}
$$

(f)

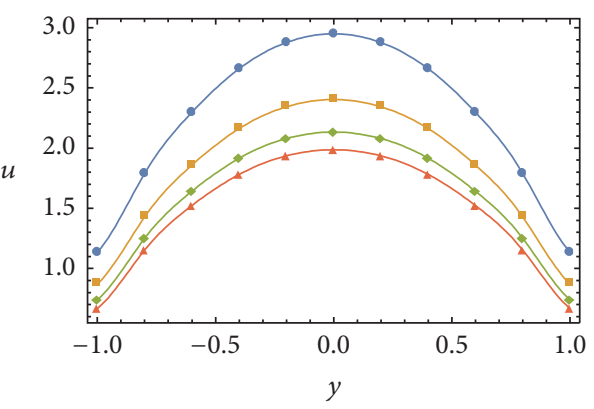

$$
\begin{aligned}
& \longrightarrow \Theta=\pi / 3 \quad \rightarrow \Theta=\pi / 5 \\
& -\Theta=\pi / 4 \quad \rightarrow \Theta=\pi / 6
\end{aligned}
$$

(h)

FIgURE 2: Variation of $\Omega, M, N, m, \alpha, F_{r}, R_{e}$, and $\Theta$ on the axial velocity $(u)$ with respect to $y$. 

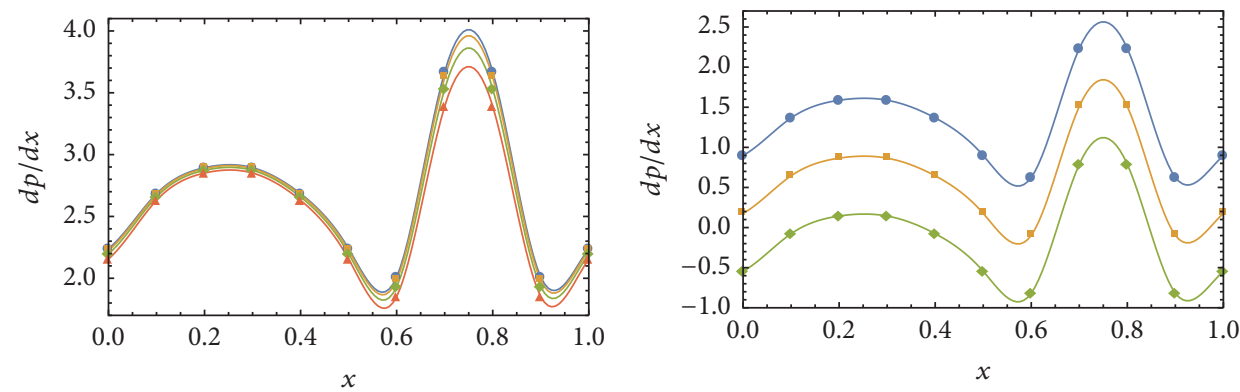

$$
\rightarrow \Omega=0.2 \quad \rightarrow \quad-\Omega=1.0
$$

(a)

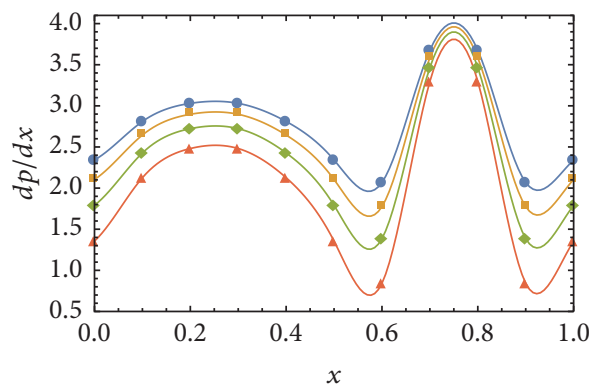

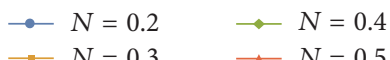

(c)

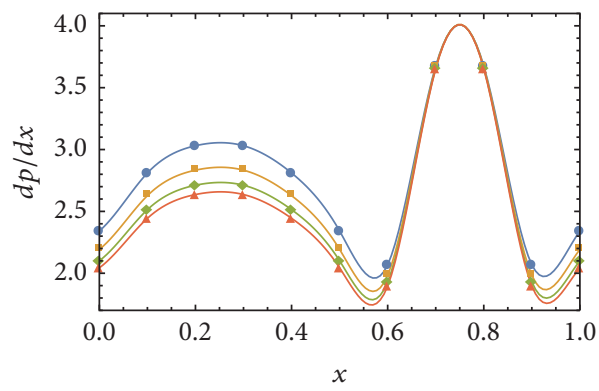

$\rightarrow F_{r}=0.15 \longrightarrow F_{r}=0.3$

$\longrightarrow F_{r}=0.2$

(b)

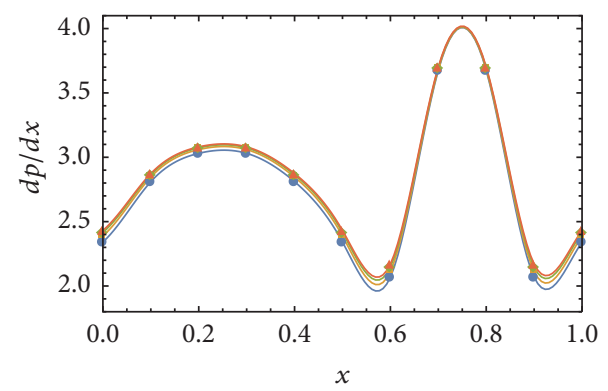

$$
\longrightarrow m=2.0 \longrightarrow m=6.0
$$$$
\because m=4.0 \leadsto m=8.0
$$

(d)

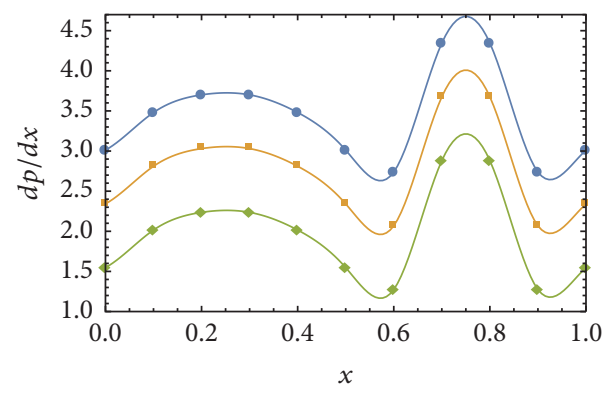

$$
\begin{aligned}
& \because \alpha=\pi / 2 \quad \rightarrow \alpha=\pi / 4 \\
& \because \alpha=\pi / 3
\end{aligned}
$$

(f)

(e)

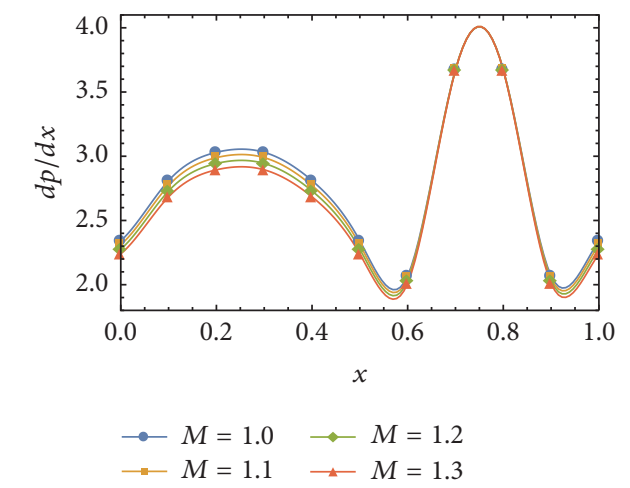

(g)

FIgURE 3: Variation of $\Omega, F_{r}, N, m, \Theta, \alpha$, and $M$ on the pressure gradient $(d p / d x)$ against $x$. 

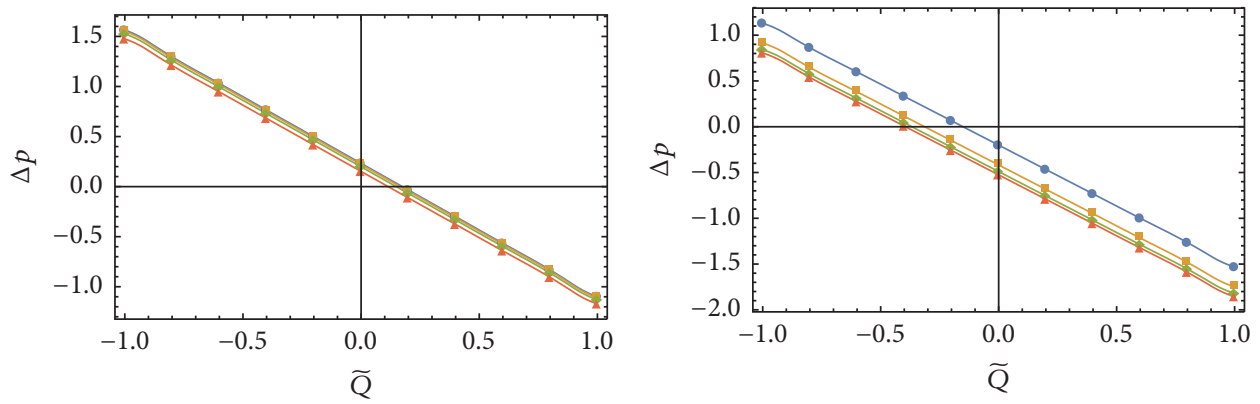

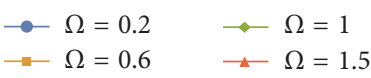

(a)

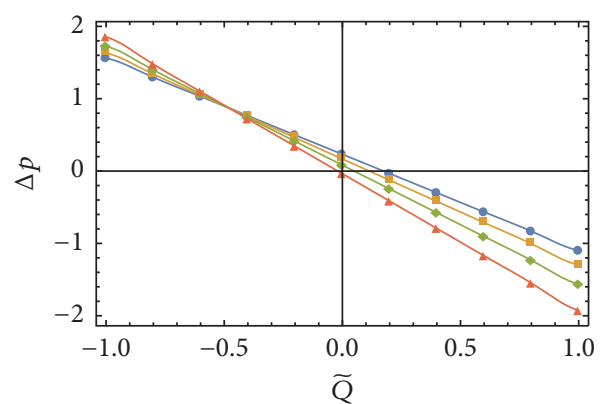

$\longrightarrow N=0.2 \quad \longrightarrow N=0.4$

$\longrightarrow N=0.3 \quad \longrightarrow N=0.5$

(c)

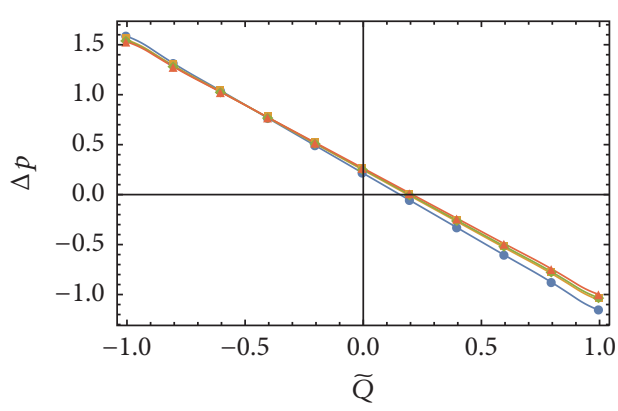

$\because m=0.1 \quad \because m=8.0$

$\because m=4.0 \quad \longrightarrow m=20$

(e)

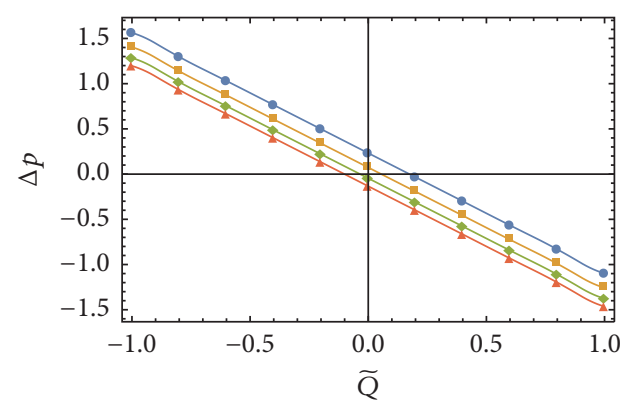

$$
\begin{aligned}
& \multimap \alpha=\pi / 3 \quad \longrightarrow \alpha=\pi / 5 \\
& \because \alpha=\pi / 4 \quad \longrightarrow \alpha=\pi / 6
\end{aligned}
$$

(g)

$$
\begin{array}{ll}
\longrightarrow F_{r}=0.2 & \longrightarrow F_{r}=0.6 \\
-F_{r}=0.4 \quad \longrightarrow F_{r}=0.8
\end{array}
$$

(b)

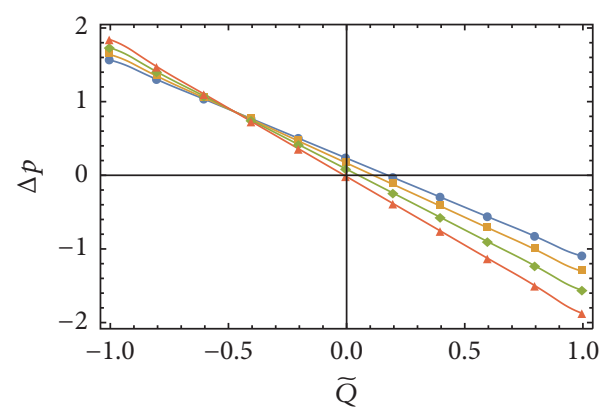

$$
\because M=1 \quad \longrightarrow M=1.6
$$

(d)

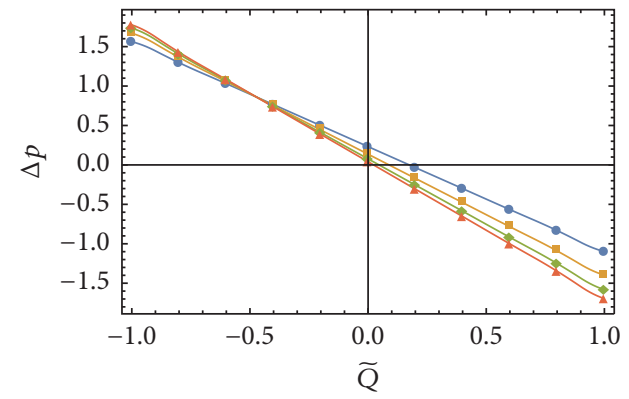

$$
\begin{aligned}
& \longrightarrow \Theta=\pi / 3 \quad \longrightarrow \Theta=\pi / 5 \\
& \because \Theta=\pi / 4 \quad \longrightarrow \Theta=\pi / 6
\end{aligned}
$$

(f)

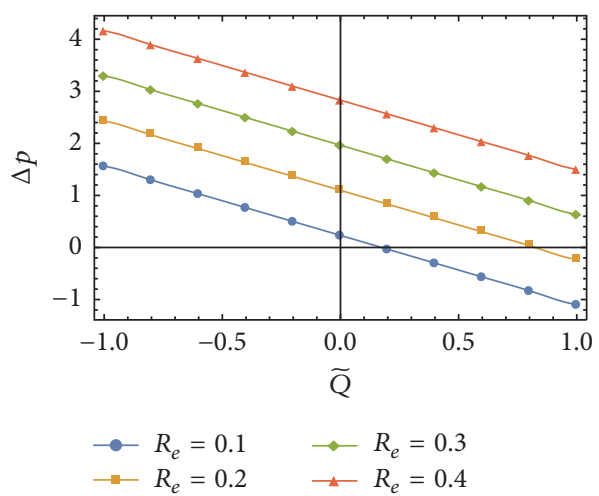

(h)

FIGURE 4: Variation of $\Omega, F_{r}, N, M, m, \Theta, \alpha$, and $R_{e}$ on the pressure rise per wavelength $(\Delta p)$ against the volume flow rate $\widetilde{Q}$. 

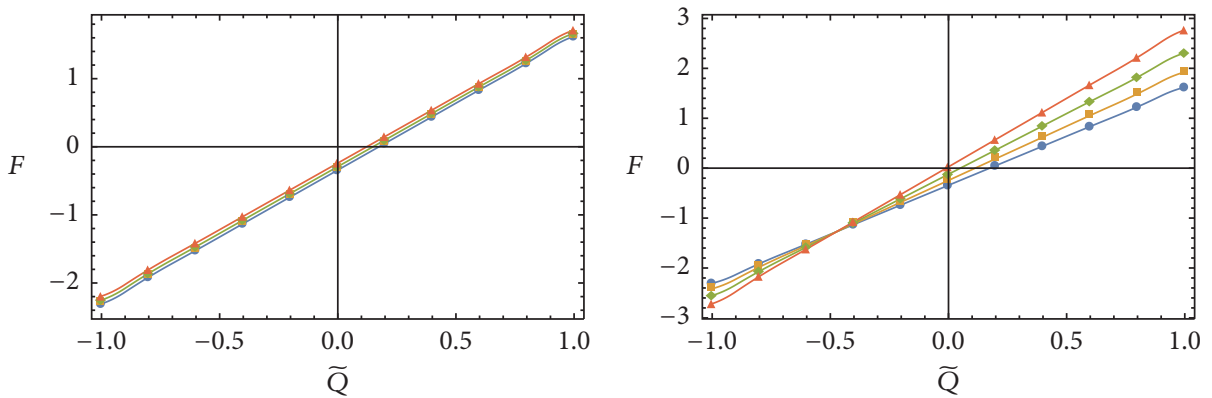

$$
\rightarrow \Omega=0.2 \quad \rightarrow \quad \Omega=1.0
$$

(a)

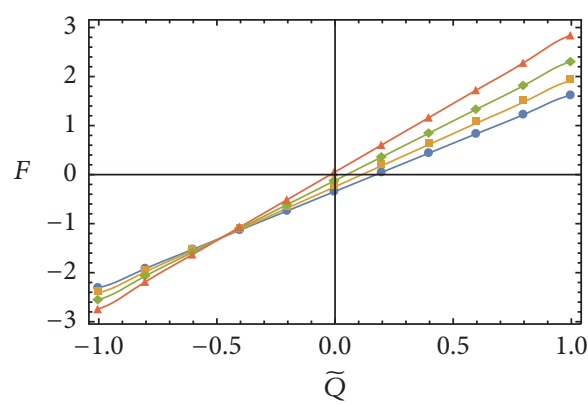

$$
\begin{aligned}
\because N=0.2 \quad \rightarrow-N & =0.4 \\
\because N=0.3 \quad \rightarrow N & =0.5
\end{aligned}
$$

(c)

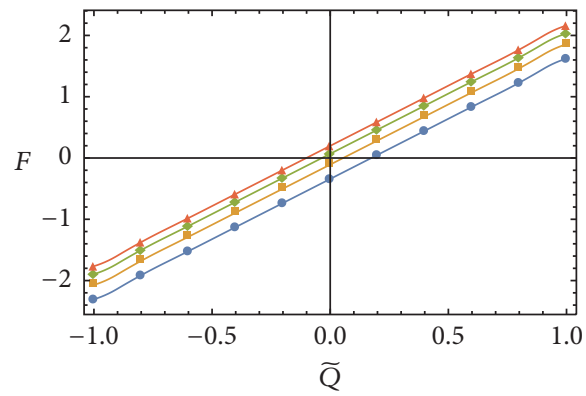

$$
\begin{aligned}
\longrightarrow \alpha & =\pi / 3 \quad \rightarrow \alpha=\pi / 5 \\
\because \alpha=\pi / 4 \quad \rightarrow \alpha & =\pi / 6
\end{aligned}
$$

(e)

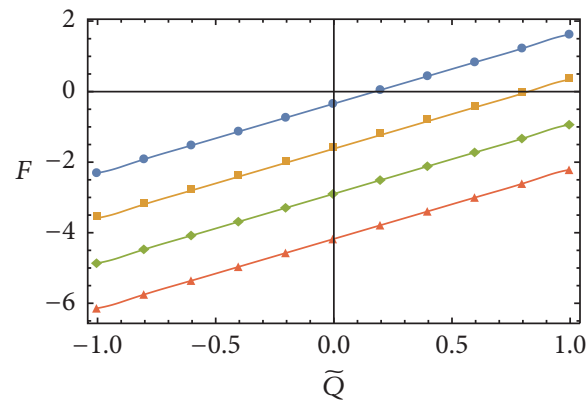

$$
\begin{array}{ll}
\longrightarrow R_{e}=0.1 \quad \longrightarrow R_{e}=0.3 \\
-R_{e}=0.2 \quad \longrightarrow R_{e}=0.4
\end{array}
$$

(g)

$$
\begin{aligned}
& \longrightarrow M=1 \quad \rightarrow M=1.6 \\
& \because M=1.3 \quad \leadsto M=1.9
\end{aligned}
$$

(b)

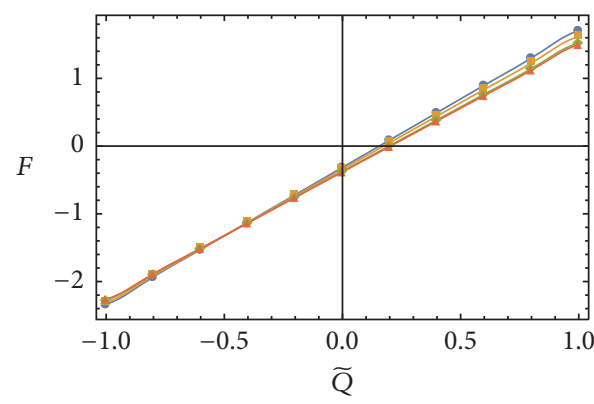

$$
\begin{aligned}
& \longrightarrow m=0.1 \quad \longrightarrow m=6.0 \\
& \because m=2.0 \quad \longrightarrow m=10
\end{aligned}
$$

(d)

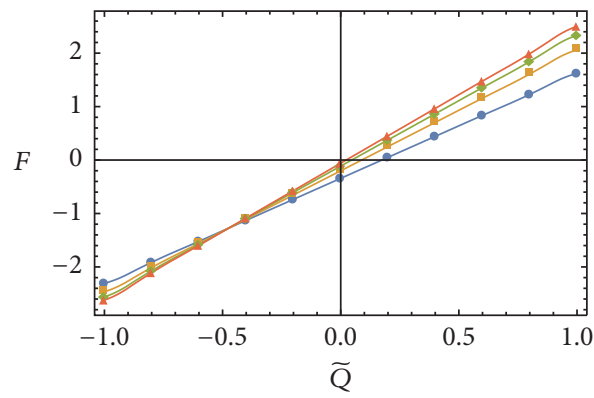

$$
\begin{aligned}
\longrightarrow \Theta & =\pi / 3 \quad \longrightarrow \Theta=\pi / 5 \\
\because \Theta & =\pi / 4 \quad \multimap \Theta=\pi / 6
\end{aligned}
$$

(f)

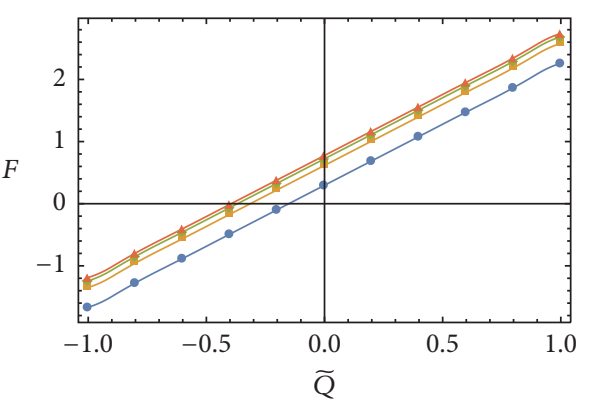

$$
\begin{aligned}
& \because F_{r}=0.2 \quad \longrightarrow F_{r}=0.6 \\
& \because F_{r}=0.4 \quad \longrightarrow F_{r}=0.8
\end{aligned}
$$

(h)

FIGURE 5: Variation of $\Omega, F_{r}, N, M, m, \Theta, \alpha$, and $R_{e}$ on the frictional force per wavelength $(F)$ against the volume flow rate $\widetilde{Q}$. 

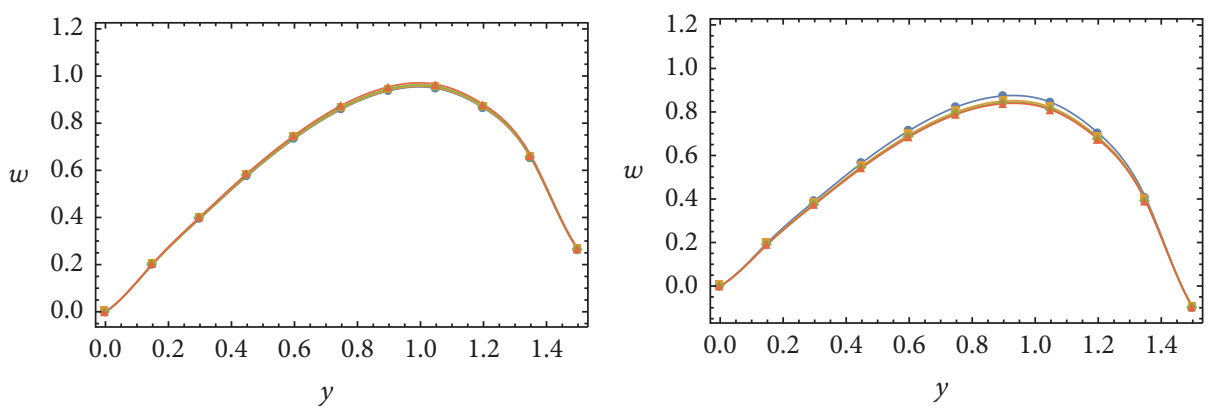

$\begin{aligned} \longrightarrow \Omega & =0.2 \\ -\Omega & =0.6\end{aligned}$

$\multimap \Omega=1.0$

$\triangle \Omega=1.4$

(a)

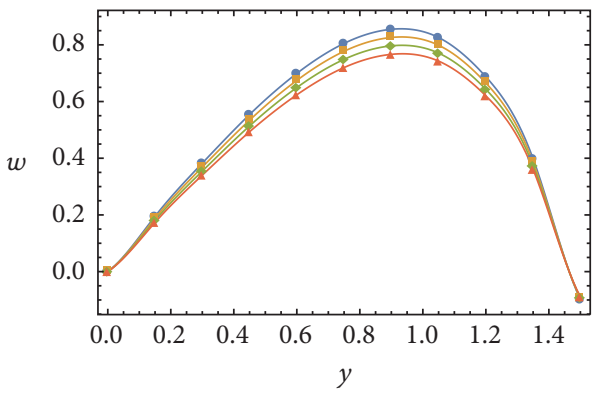

$\longrightarrow M=1.0$

$-M=1.1$

(c)

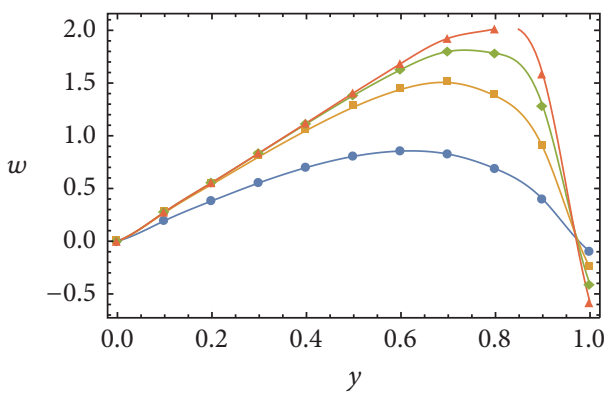

$\longrightarrow m=2.0$

$\neg m=6.0$

$-m=4.0$

$\neg m=8.0$

(e)

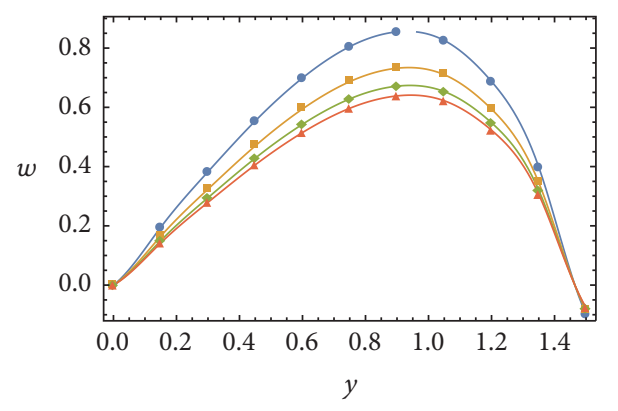

$\begin{array}{ll}\because \Theta & =\pi / 3 \\ \because \Theta & =\pi / 4 \quad \rightarrow \Theta=\pi / 5 \\ \because \Theta & =\pi / 6\end{array}$

(g)

$$
\begin{array}{ll}
\multimap F_{r}=0.3 & \rightarrow F_{r}=0.9 \\
-F_{r}=0.6 & \rightarrow F_{r}=1.2
\end{array}
$$

(b)

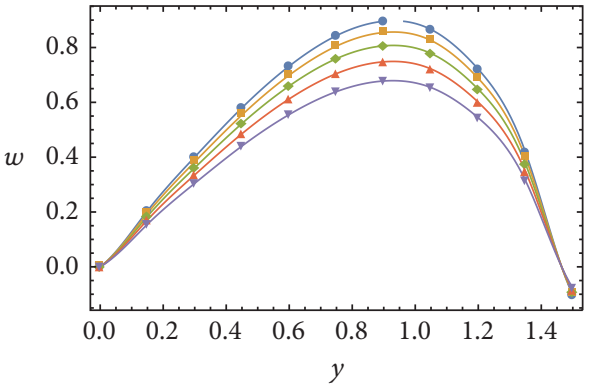

$$
\rightarrow N=0.1 \rightarrow N=0.3 \rightarrow N=0.5
$$

(d)

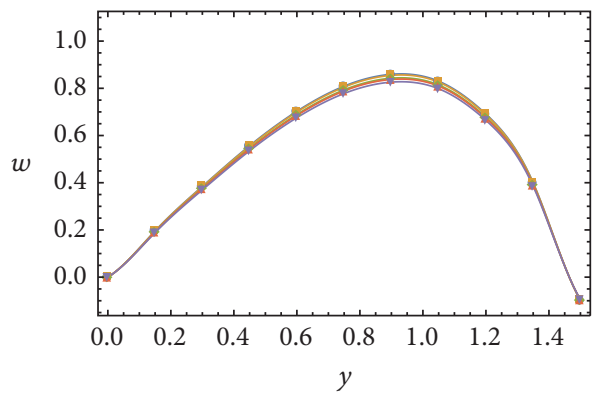

$$
\rightarrow \alpha=\pi / 2 \rightarrow \alpha=\pi / 6 \rightarrow \alpha=0
$$$$
\square \alpha=\pi / 3 \quad \longleftarrow \alpha=\pi / 9
$$

(f)

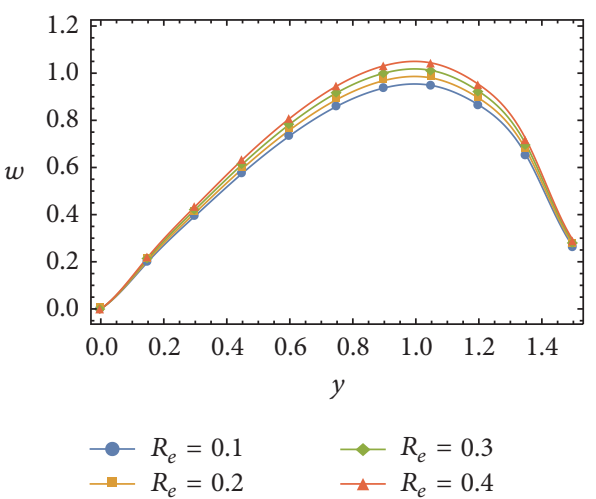

(h)

FIGURE 6: Variation of $\Omega, F_{r}, M, N, m, \alpha, \Theta$, and $R_{e}$ on the spin velocity $(w)$ with respect to $y$. 

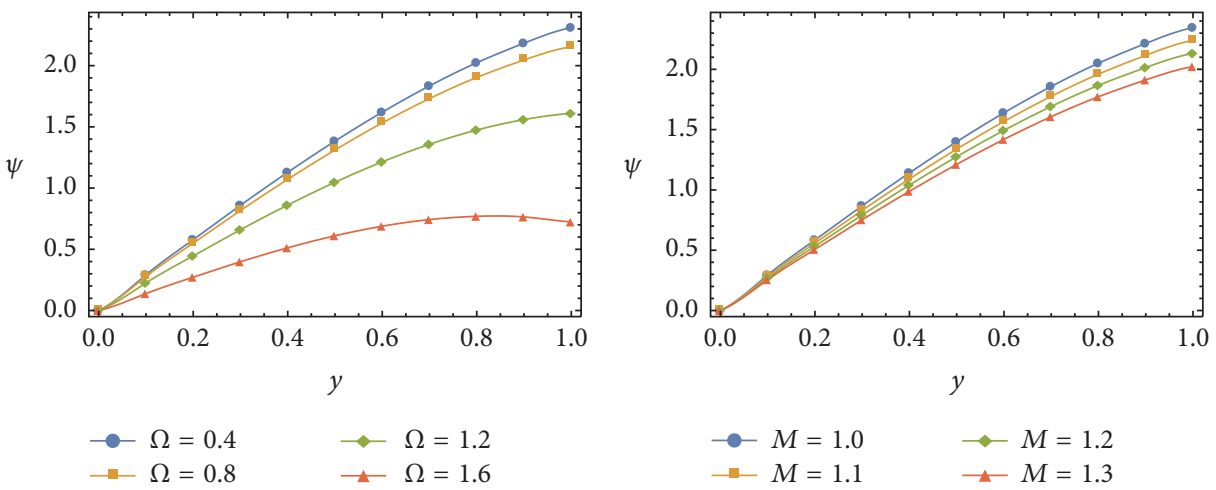

(a)
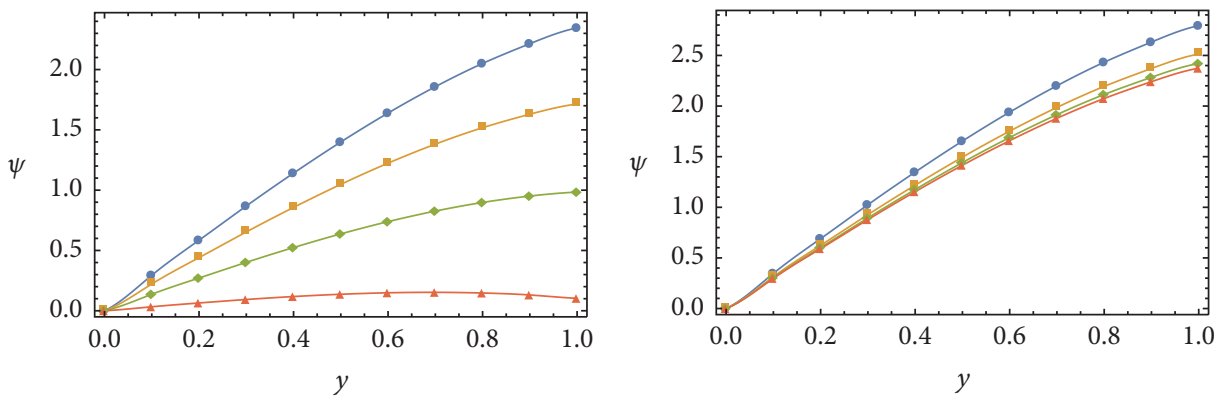

$$
\begin{aligned}
\longrightarrow N=0.2 & \longrightarrow N=0.6 \\
-N=0.4 & \longleftarrow N=0.8
\end{aligned}
$$

(c)

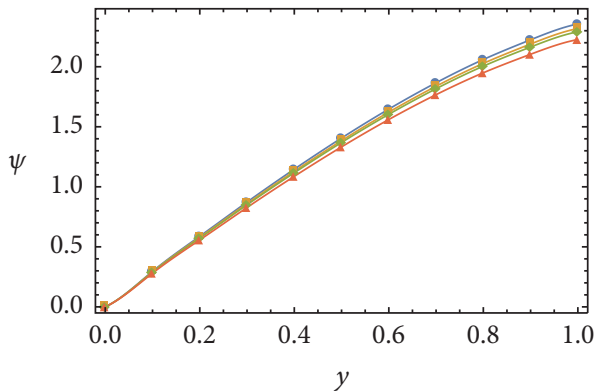

$$
\begin{array}{ll}
\longrightarrow \alpha=\pi / 3 & \longrightarrow \alpha=\pi / 6 \\
\longrightarrow \alpha=\pi / 4 & \rightarrow \alpha=0
\end{array}
$$

(e)

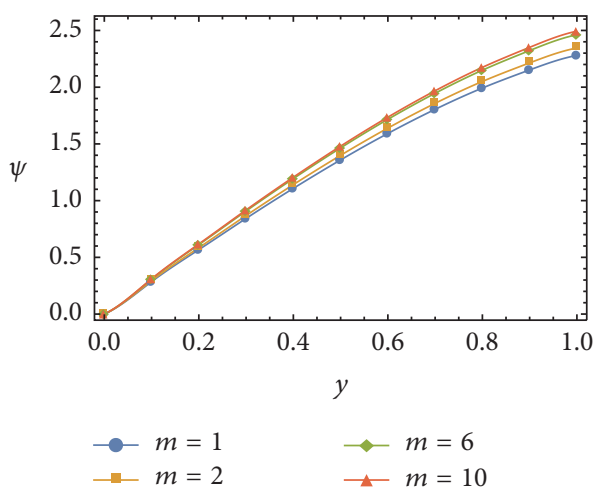

(g)

$$
\begin{aligned}
& \longrightarrow F_{r}=0.1 \quad \rightarrow F_{r}=0.3 \\
& \because-F_{r}=0.2 \quad \rightarrow F_{r}=0.4
\end{aligned}
$$

(d)

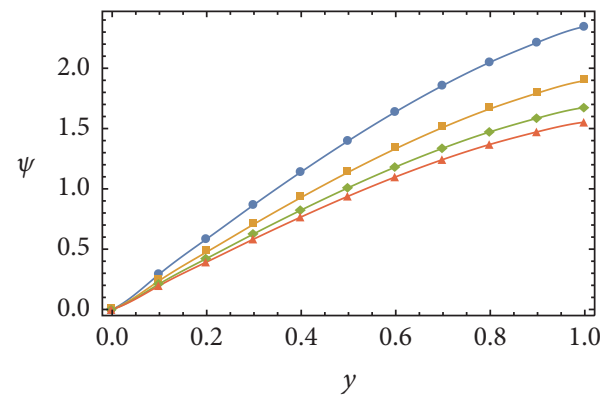

$$
\rightarrow \Theta=\pi / 3 \quad \rightarrow-\Theta=\pi / 5
$$

(f)

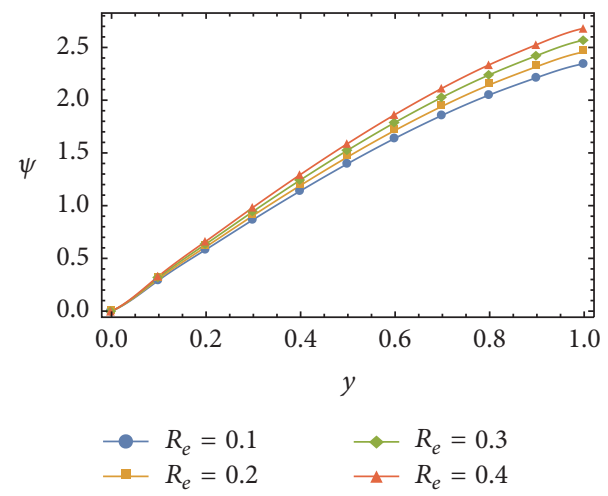

(h)

Figure 7: Variation of $\Omega, M, N, F_{r}, \alpha, \Theta, m$, and $R_{e}$ on the stream function $(\psi)$ with respect to $y$. 
region $(\Delta p<0, \widetilde{\mathrm{Q}}>0)$, and free pumping region $(\Delta p=0)$. The region in which $(\Delta p>0, \widetilde{Q}>0)$ is known as the peristaltic pumping region. In this region the peristaltic wave overwhelms the pressure rise and propagates the fluid in the direction of its propagation. The region in which $\Delta p>0$ and $\widetilde{Q}<0$ is called a retrograde pumping region. In this locale, the stream is inverse to the course of the peristaltic movement. The region, in which $(\Delta p<0, \widetilde{Q}>0)$, is known as augmented or copumping region. In this region, the negative pressure rise enhances the flow due to the peristalsis of the walls. In the free pumping region, where $\Delta p=0$, the flow is totally prompted by the peristalsis of the walls.

From Figures 4(a)-4(b) it is observed that the pressure rise per wavelength decreases with increasing rotation and Froude number. In Figures 4(c)-4(d), it is clear that with increase in the coupling number and magnetic field parameter the pumping rate increases up to a critical value of $(\widetilde{Q})$ and decreases after a critical value of $(\widetilde{Q})$ in the retrograde pumping region while it decreases in the peristaltic, free, and copumping regions. Figures 4(e)-4(f) illustrate that the micropolar parameter and inclination angle of the magnetic field parameter have opposite behavior as compared to the coupling number $(N)$ and magnetic field parameter $(M)$. Figures $4(\mathrm{~g})-4(\mathrm{~h})$ show that inclination angle of the channel $(\alpha)$ and Reynold number $\left(R_{e}\right)$ exactly have an inverse behavior when contrasted with the rotation $(\Omega)$ and Froude number $\left(F_{r}\right)$. Figures $5(\mathrm{a})-5(\mathrm{~h})$ describe the variation of frictional forces against the volume flow rate for diverse rising parameters. The frictional forces precisely have an inverse conduct when contrasted with the pressure rise.

Figures $6(\mathrm{a})-6(\mathrm{~h})$ represent the variation of spin velocity $(w)$ for different parameters of interest. It is clear from Figure 6(a) that the effect of rotation on the spin velocity is negligible. From Figures 6(b)-6(d) it is observed that spin velocity decreases with increasing Froude number $\left(F_{r}\right)$, magnetic field parameter $(M)$, and the coupling number $(N)$. Figures $6(\mathrm{e})-6(\mathrm{~h})$ illustrate that the spin velocity increases with increasing micropolar parameter $(m)$, inclination angle of the channel $(\alpha)$, inclination angle of the magnetic field parameter $(\Theta)$, and Reynold number $\left(R_{e}\right)$. Figures $7(\mathrm{a})-7(\mathrm{~h})$ describe the variation of stream function pertinent to different physical parameters. It is illustrated from Figures 7(a)$7(\mathrm{~d})$ that the stream function $(\psi)$ decreases with increasing the rotation $(\Omega)$, magnetic field parameter $(M)$, coupling number $(N)$, and Froude number $\left(F_{r}\right)$. It is also observed that the effect of $(m),(\alpha),(\Theta)$, and $\left(R_{e}\right)$ on the stream function is totally opposite when compared with rotation $(\Omega)$, magnetic field parameter $(M)$, coupling number $(N)$, and Froude number $\left(F_{r}\right)$. From Figures $7(\mathrm{e})-7(\mathrm{~h})$, it is clear that the stream function increments with expanding inclination angle of the channel $(\alpha)$, inclination angle of the magnetic field parameter $(\Theta)$, micropolar parameter $(m)$, and Reynold number $\left(R_{e}\right)$.

\section{Conclusion}

The present investigation discusses the rotation and inclined magnetic field effects on the peristaltic flow of a micropolar fluid in an inclined channel. The main findings can be summarized below:

(i) The velocity profile increases in view of an increase in $m, R_{e}, \Theta$, and $\alpha$ but decreases with increasing $\Omega, M$, $N$, and $F_{r}$.

(ii) Pressure gradient increases by increasing $m$ and $\Theta$ in the wider part of the channel while in the narrow part of the channel there is no appreciable difference.

(iii) The pumping rate increases by increasing the inclination angle of the channel.

(iv) The magnetic field improves the pumping rate.

(v) $\Delta p$ increases entirely with an increase in the inclination angle of the channel $\alpha$ and Reynolds number $R_{e}$ while rotation $\Omega$ and Froude number $F_{r}$ have opposite behavior on $\Delta p$ when compared with $\alpha$ and $R_{e}$.

(vi) The effects of $M$ and $\Theta$ on the pressure rise are qualitatively opposite.

(vii) The frictional forces have an opposite behavior as compared to pressure rise.

(viii) The qualitative effects of $M, N$, and $F_{r}$ on the spin velocity are similar.

(ix) The effect of $\alpha$ and $\Theta$ on spin velocity is exactly opposite when compared with $N$ and $M$.

(x) The stream function decreases with increasing $\Omega, M$, and $N$ while it increases with increasing $m, \alpha$, and $\Theta$.

\section{Competing Interests}

The authors declare that they have no competing interests.

\section{References}

[1] T. W. Latham, Fluid Motion in a Peristaltic Pump, Massachusetts Institute of Technology, Cambridge, UK, 1966.

[2] K. S. Mekheimer, "Peristaltic transport of a couple-stress fluid in a uniform and nonuniform channels," Biorheology, vol. 39, pp. 755-765, 2002.

[3] E. F. Elshehawey, N. T. Eldabe, E. M. Elghazy, and A. Ebaid, "Peristaltic transport in an asymmetric channel through a porous medium," Applied Mathematics and Computation, vol. 182, no. 1, pp. 140-150, 2006.

[4] T. Hayat, Q. Hussain, and N. Ali, "Influence of partial slip on the peristaltic flow in a porous medium," Physica A. Statistical Mechanics and Its Applications, vol. 387, no. 14, pp. 3399-3409, 2008.

[5] T. Hayat, N. Ali, S. Asghar, and A. M. Siddiqui, "Exact peristaltic flow in tubes with an endoscope," Applied Mathematics and Computation, vol. 182, no. 1, pp. 359-368, 2006.

[6] A. H. Abd El Naby and A. E. M. El-Misiery, "Effects of an endoscope and generalized Newtonian fluid on peristaltic motion," Applied Mathematics and Computation, vol. 128, no. 1, pp. 19-35, 2002.

[7] P. Hariharan, V. Seshadri, and R. K. Banerjee, "Peristaltic transport of non-newtonian fluid in a diverging tube with different wave forms," Mathematical and Computer Modelling, vol. 48, no. 7-8, pp. 998-1017, 2008. 
[8] A. C. Eringen, “Theory of micropolar fluids," Journal of Applied Mathematics and Mechanics, vol. 16, pp. 1-16, 1966.

[9] K. S. Mekheimer, "Peristaltic flow of a magneto-micropolar fluid: effect of induced magnetic field," Journal of Applied Mathematics, vol. 2008, Article ID 570825, 23 pages, 2008.

[10] S. K. Pandey and M. K. Chaube, "Peristaltic flow of a micropolar fluid through a porous medium in the presence of an external magnetic field," Communications in Nonlinear Science and Numerical Simulation, vol. 16, no. 9, pp. 3591-3601, 2011.

[11] V. C. A. Ferraro and C. Plumpton, An Introduction to Magneto Fluid Mechanics, 1966.

[12] V. K. Sud, G. S. Sekhon, and R. K. Mishra, "Pumping action on blood by a magnetic field," Bulletin of Mathematical Biology, vol. 39, no. 3, pp. 385-390, 1977.

[13] P. Wlodarczyk, L. Hawelek, M. Paluch, A. Wlodarczyk, Z. Wojnarowska, and A. Kolano-Burian, "Dielectric properties of glassy disaccharides for electromagnetic interference shielding application," Journal of Applied Physics, vol. 118, no. 18, Article ID 184102, 2015.

[14] E. Calabrò, S. Condello, S. Magazù, and R. Ientile, "Static and $50 \mathrm{~Hz}$ electromagnetic fields effects on human neuronal-like cells vibration bands in the mid-infrared region," Journal of Electromagnetic Analysis and Applications, vol. 3, no. 2, pp. 6978, 2011.

[15] E. Calabrò, Effects of Electromagnetic Fields on Cells and Proteins Structure, Lambert Academy, Saarbrücken, Germany, 2012.

[16] T. Hayat, N. Alvi, and N. Ali, "Peristaltic mechanism of a Maxwell fluid in an asymmetric channel," Nonlinear Analysis: Real World Applications, vol. 9, no. 4, pp. 1474-1490, 2008.

[17] A. E. H. Abd El Naby, A. E. M. El Misery, and M. F. Abd El Kareem, "Effects of a magnetic field on trapping through peristaltic motion for generalized Newtonian fluid in channel," Physica A: Statistical Mechanics and Its Applications, vol. 367, pp. 79-92, 2006.

[18] M. Mishra and A. R. Rao, "Peristaltic transport of a Newtonian fluid in an asymmetric channel," Zeitschrift für Angewandte Mathematik und Physik, vol. 54, no. 3, pp. 532-550, 2003.

[19] S. Srinivas and M. Kothandapani, "The influence of heat and mass transfer on MHD peristaltic flow through a porous space with compliant walls," Applied Mathematics and Computation, vol. 213, no. 1, pp. 197-208, 2009.

[20] S. Nadeem and N. S. Akbar, "Influence of radially varying MHD on the peristaltic flow in an annulus with heat and mass transfer," Journal of the Taiwan Institute of Chemical Engineers, vol. 41, no. 3, pp. 286-294, 2010.

[21] A. M. Abd-Alla, G. A. Yahya, S. R. Mahmoud, and H. S. Alosaimi, "Effect of the rotation, magnetic field and initial stress on peristaltic motion of micropolar fluid," Meccanica, vol. 47, no. 6, pp. 1455-1465, 2012.

[22] S. R. Mahmoud, A. M. Abd-Alla, and M. A. El-Sheikh, "Effect of the rotation on wave motion through cylindrical bore in a micropolar porous medium," International Journal of Modern Physics B, vol. 25, no. 20, pp. 2713-2728, 2011.

[23] D. Srinivasacharya, M. Mishra, and A. R. Rao, "Peristaltic pumping of a micropolar fluid in a tube," Acta Mechanica, vol. 161, no. 3-4, pp. 165-178, 2003.

[24] B. Mohanty, S. R. Mishra, and H. B. Pattanayak, "Numerical investigation on heat and mass transfer effect of micropolar fluid over a stretching sheet through porous media," Alexandria Engineering Journal, vol. 54, no. 2, pp. 223-232, 2015.
[25] A. M. Abd-Alla, S. R. Mahmoud, and N. A. Al-Shehri, "Effect of the rotation on a non-homogeneous infinite cylinder of orthotropic material," Applied Mathematics and Computation, vol. 217, no. 22, pp. 8914-8922, 2011.

[26] A. M. Abd-Alla, S. R. Mahmoud, S. M. Abo-Dahab, and M. I. Helmy, "Influences of rotation, magnetic field, initial stress, and gravity on Rayleigh waves in a homogeneous orthotropic elastic half-space," Applied Mathematical Sciences, vol. 4, no. 2, pp. 91108, 2010.

[27] A. M. Abd-Alla and S. R. Mahmoud, "Magneto-thermoelastic problem in rotating non-homogeneous orthotropic hollow cylinder under the hyperbolic heat conduction model," Meccanica, vol. 45, no. 4, pp. 451-462, 2010.

[28] A. M. Abd-Alla and S. R. Mahmoud, "Effect of rotation on thermoelastic waves in a non-homogeneous infinite cylinder," International Journal of Mathematical Analysis, vol. 4, no. 4144, pp. 2051-2064, 2010.

[29] B. Punnamchandar and T. K. V. Iyengar, "Pulsating flow of an incompressible micropolar fluid between permeable beds with an inclined uniform magnetic field," European Journal of Mechanics-B/Fluids, vol. 48, pp. 174-182, 2008.

[30] K. Ramesh and M. Devakar, "Magnetohydrodynamic peristaltic transport of couple stress fluid through porous medium in an inclined asymmetric channel with heat transfer," Journal of Magnetism and Magnetic Materials, vol. 394, pp. 335-348, 2015.

[31] D. Srinivasacharya and K. Hima Bindu, "Entropy generation in a micropolar fluid flow through an inclined channel with slip and convective boundary conditions," Energy, vol. 91, pp. 72-83, 2015.

[32] M. M. Rahman, A. Aziz, and M. A. Al-Lawatia, "Heat transfer in micropolar fluid along an inclined permeable plate with variable fluid properties," International Journal of Thermal Sciences, vol. 49, no. 6, pp. 993-1002, 2010.

[33] K. Das, "Effect of chemical reaction and thermal radiation on heat and mass transfer flow of MHD micropolar fluid in a rotating frame of reference," International Journal of Heat and Mass Transfer, vol. 54, no. 15-16, pp. 3505-3513, 2011.

[34] M. M. Khonsari and D. E. Brewe, "Effect of viscous dissipation on the lubrication characteristics of micropolar fluids," Acta Mechanica, vol. 105, no. 1-4, pp. 57-68, 1994.

[35] A. M. Abd-Alla, S. M. Abo-Dahab, and R. D. Al-Simery, "Effect of rotation on peristaltic flow of a micropolar fluid through a porous medium with an external magnetic field," Journal of Magnetism and Magnetic Materials, vol. 348, pp. 33-43, 2013. 

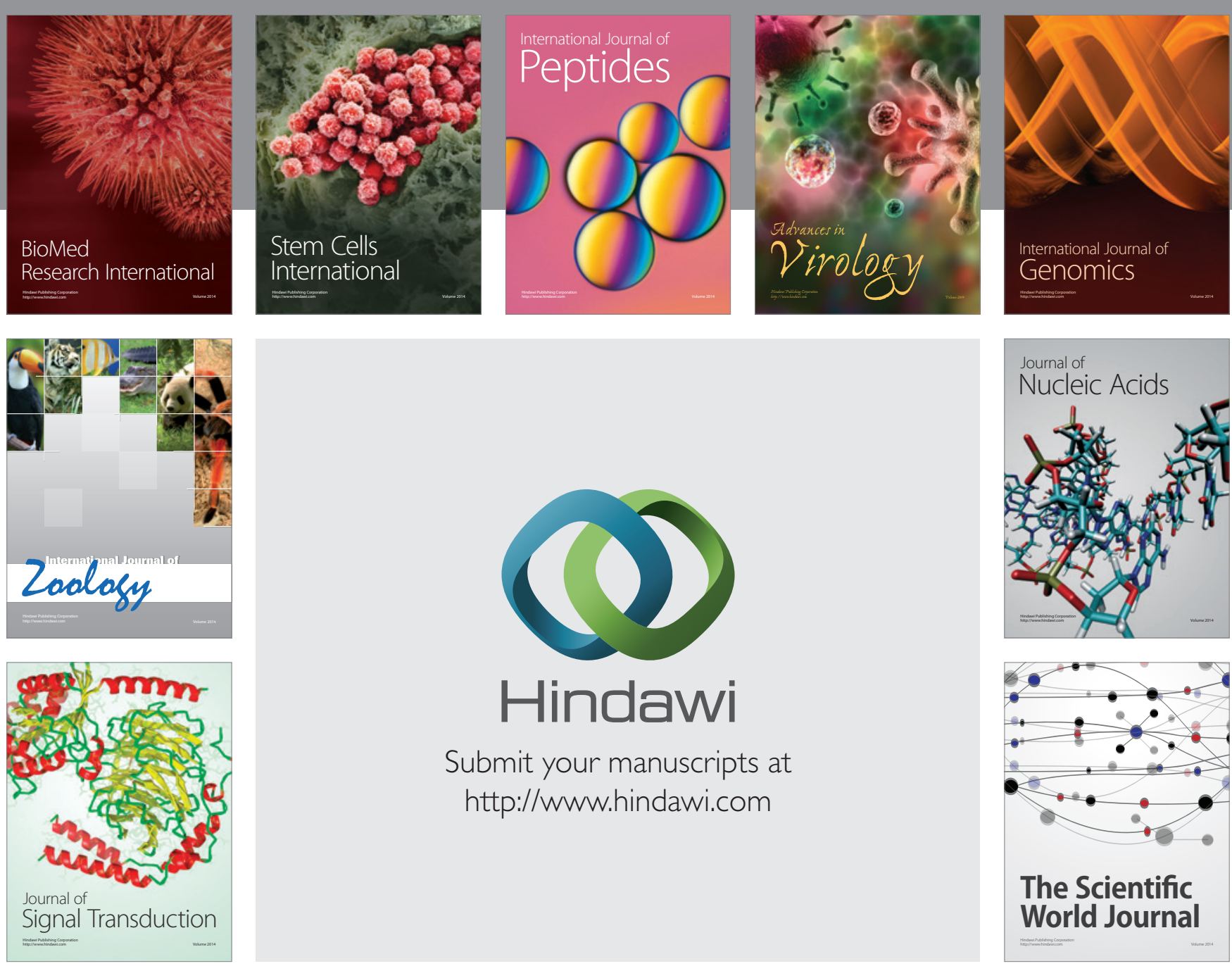

Submit your manuscripts at

http://www.hindawi.com
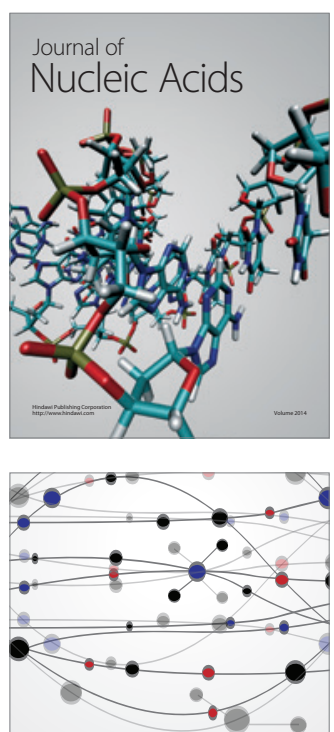

The Scientific World Journal
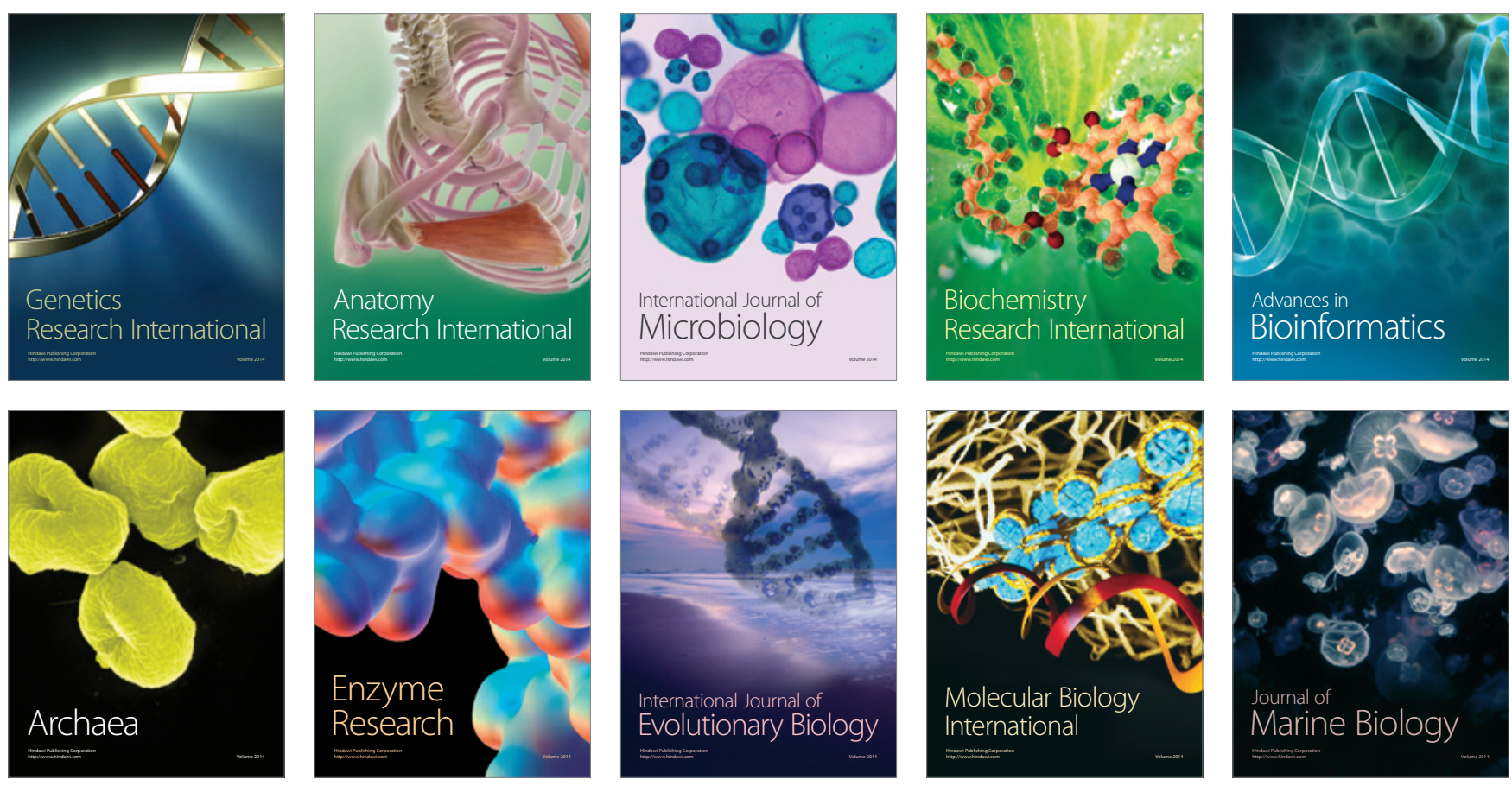